\title{
NEW LIGHT ON UU SAGITTAE
}

\author{
S.A. BELL and D.L. POLLACCO
}

Department of Physics and Astronomy, North Haugh, St. Andrews, Fife KY16 9SS, Scotland

New $\mathrm{V}$ and I band CCD photometry and medium resolution spectroscopy are used to derive the masses, luminosities and radii accurate to $<10 \%$ for the individual components of the eclipsing central star of the planetary nebula A63UU Sge $\left(\mathrm{M}_{1}=0.63 \pm 0.06 \mathrm{M}_{\odot}, \mathrm{R}_{1}=0.33 \pm 0.01 \mathrm{R}_{\odot}, \mathrm{M}_{2}=0.29 \pm 0.04 \mathrm{M}_{\odot}\right.$ and $\left.R_{2}=0.53 \pm 0.02 R_{\odot}\right)$. Emission lines from the secondary component and HeII and $\mathrm{NV}$ absorption features from the primary component are used to determine the first radial velocity curves of the system. Ultra-violet and optical spectra show that the temperature of the primary compoment is $\sim 10^{5} \mathrm{~K}$ - much larger than previously suspected. As the techniques used are essentially independent this is probably the most accurately known mass for a planetary nebula central star and therefore allows meaningful comparison to be made with evolutionary tracks for these objects. 